\title{
Silastic rod encirclement in retinal detachment surgery
}

\author{
AMIN ZAYED, BAHY SHALASH, AND PIERRE AMALRIC \\ From the Department of Ophthalmology, Cairo University, and the Ophthalmic Centre, Albi, France
}

Various techniques have been described for the surgical repair of retinal detachment. In the forefront of retinal detachment surgery, stands the encircling operation. A variety of materials have been utilized to create a permanent annular indentation of the sclera.

In this communication we report our experience with silastic rod cerclage for the treatment of retinal detachment. Silastic is white inert silicone sponge. It is soft, elastic, and compressible, and can be readily sterilized by dry heat in a hot-air oven. It is available in cylindrical forms with diameters varying from I. 5 to $7 \mathrm{~mm}$.

\section{Materials and methods}

Silastic rod encirclement was performed in 200 cases of rhegmatogenous retinal detechment.

Three techniques were adopted for the cases reported below (Table I):

\section{Table I Surgical procedures}

\begin{tabular}{lll}
\hline Operation & No. of cases \\
${$\cline { 1 - 1 }$} }$ & $7 \mathrm{I}$ \\
Silastic rod cerclage+circumferential buckle & 75 \\
Silastic rod cerclage+meridional buckle & & 54 \\
\hline Total cases & & 200
\end{tabular}

(r) Simple silastic rod encirclement, using a $3 \mathrm{~mm}$. rod, was performed in 71 cases of detachment, all associated with multiple small or medium-sized breaks, lying at or peripheral to the equator (Fig. I).

(2) Silastic rod encirclement combined with an additional circumferential silastic implant was used in 75 cases which had, in addition to multiple peripheral breaks, a large break with the longest axis lying parallel to the limbus (Fig. 2).

(3) Silastic rod encirclement combined with a meridional silastic implant was used in 54 cases which had, in addition to multiple peripheral breaks, either a large break with its long axis perpendicular to the limbus, or a break which lay well behind the equator of the globe (Fig. 3).

Address for reprints: Asst. Prof. Amin Zayed, 84 Abou Bakr Al Saddik, Heliopolis, Cairo, Egypt

\section{SURGICAL PROCEDURE}

The retinal breaks are localized by scleral indentation under continuous observation with the indirect ophthalmoscope. The posterior edge of the most posterior break is marked with gentian violet.

The retinal breaks are then surrounded with confluent cryoapplications. Mattress anchoring scleral sutures are placed at least one in each quadrant. The scleral sutures over the silastic rod are tied with temporary knots. More scleral sutures, also with temporary knots, may be inserted if there is an indication for the use of an additional circumferential or meridional silastic implants.

The fundus is then examined with a binocular indirect ophthalmoscope to check the break-to-buckle relationship, as well as the patency of the central retinal artery. The surgeon then proceeds as follows:

(I) If the buckle is recognized to be in a faulty position, the overlying scleral sutures are cut and the buckle is readjusted to cover the retinal breaks.

(2) If the buckle lies in the proper place, the retinal breaks are close to its summit, and the central retinal artery is patent, the subretinal fluid is not drained, but is left for spontaneous absorption which is usually achieved within 24 to $48 \mathrm{hrs}$ after the operation.

(3) If the buckle is located in the proper place, the retinal breaks are close to its summit, and the central retinal artery is occluded, an immediate paracentesis is done to restore the arterial pulsations, and the subretinal fluid is not drained.

(4) If the buckle lies in the proper place and the retinal breaks are lifted off the buckle as occurs in ballooned detachment, the subretinal fluid is drained.

Additional indications for fluid drainage are set out in Table II.

Table II Indications for fluid drainage

\section{Indication}

No. of cases

Bullous detachment

Long-standing detachment

Posterior breaks

Macular holes

Co-existing glaucoma

Co-existing retinitis pigmentosa

Total cases

$\begin{array}{r}42 \\ 24 \\ 23 \\ 6 \\ 7 \\ 2 \\ \hline\end{array}$

104 


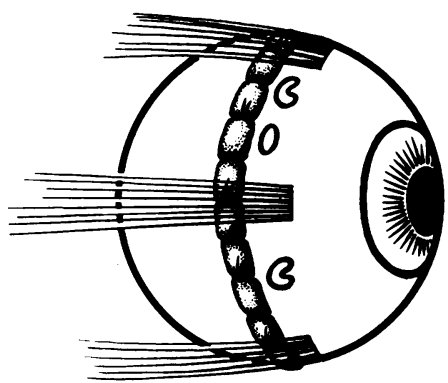

I

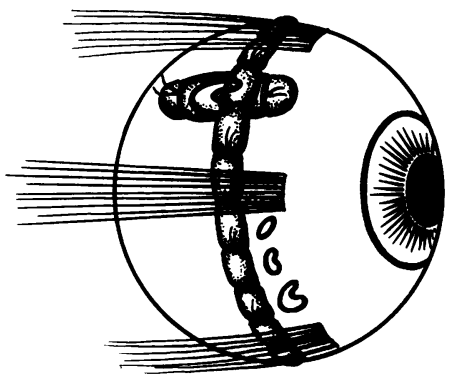

3

The subretinal fluid was not drained in 96 cases, and was drained in 104 cases; in 26 of the latter, an intravitreal injection of saline or air was given to combat ocular hypotony, to flatten meridional retinal folds which appeared across the annular buckle and to assist the closure of those breaks which failed to be apposed to the underlying buckle despite the release of subretinal fluid.

The intravitreal injection was given just anterior and to one side of the inferior rectus muscle insertion in order to be sure that the vitreous was entered rather than a syneresis pocket. The tip of the needle was seen to be free in the vitreous before the injection was attempted. The injection was given slowly with repeated testing of the intraocular pressure with a sterile tonometer held perpendicular to the cornea. The injection was terminated when the apposition of the breaks to the buckle was achieved and the intraocular pressure was below normal to allow for adjustment of the encircling rod.

\section{Results}

The retina was successfully re-attached in ${ }^{1} 64$ cases (82 per cent.). This may be considered satisfactory, in view of the unfavourable condition of the detached retina before the encirclement operation. III.

The postoperative complications are listed in Table

Choroidal detachment (8 cases) occurred only in cases in which the subretinal fluid was drained. The choroidal detachment absorbed spontaneously in all cases.

In the four cases of postoperative infection, the wound was reopened and irrigated with the appropriate antibiotic, and the silastic and retaining sutures

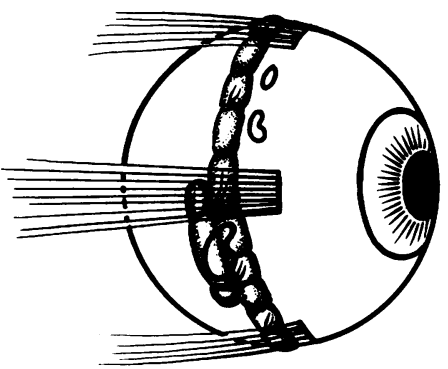

2

FIG. I Silastic rod encirclement in a case of detachment with multiple peripheral breaks

FIG. 2 Silastic rod encirclement with an additional circumferential silastic implant covering a large break with the longest axis parallel to the limbus

FIG. 3 Silastic rod encirclement with an additional meridional implant covering a large break with the longest axis perpendicular to the limbus

Table III Postoperative complications

\begin{tabular}{lc}
\hline Complication & No. of cases \\
\cline { 2 - 2 } Choroidal detachment & 8 \\
Infection & 4 \\
Uveitis & 12 \\
Spontaneous extrusion of silastic & 4 \\
Subconjunctival presentation of silastic & 20
\end{tabular}

were removed. Three eyes were saved but one was lost. Two of the three eyes which were saved by removal of the silastic had recurrence of the detachment, because the buckle was removed too early, before the formation of water-tight adhesions around the retinal breaks.

There were twelve cases of postoperative uveitis. Systemic corticosteroid therapy was effective in suppressing the inflammation; in two cases the silastic was removed, but fortunately without recurrence of the detachment.

Dehiscence of the overlying conjunctiva with extrusion of the silastic was observed in four cases, in all of which the silastic implants were $5 \mathrm{~mm}$. in diameter or more, and the implant was placed radially.

Subconjunctival presentation of the silastic, with an intact overlying conjunctiva, was seen in twenty cases, which were left alone.

Anterior segment necrosis did not occur, probably because cryopexy was used instead of diathermy, and 
the rectus muscles were not tenotomized. The former saves the posterior ciliary arteries, and the latter the anterior ciliary arteries.

Intraocular erosion of the encircling silastic rod has not occurred in a follow-up period ranging from 6 months to 4 years. Erosion was probably avoided by the soft and compressible character of the silastic. Moreover the underlying sclera is not undermined by scleral dissection or weakened by diathermy applications.

\section{Discussion}

The encircling operation is indicated for cases of retinal detachment associated with marked degree of vitreous traction, multiple peripheral breaks lying in different meridia, subtotal detachments with extensive degenerative areas in the remaining attached retina, as well as for aphakic cases.

Silastic rod encirclement was reported by (Chawla, 1971), who used a $7 \mathrm{~mm}$. silastic sponge, halved lengthwise, to produce a broad shallow implant, the subretinal fluid being released as completely as possible through a meridional scratch incision.

The following year Mortada (1972) reported using a $3 \mathrm{~mm}$. silastic rod in 100 cases of detachment without evacuation of subretinal fluid.

Our technique differs from these in two respects:

(I) The encircling rod is supplemented, if necessary, with circumferential or meridional silastic sponge implants in order to obtain a more localized buckling effect at the site of the retinal break. The additional local implant can be tailored to cover big breaks, as well as those which lie in awkward positions and cannot easily be sealed with an encircling rod. The combination of encircling rod with segmental implant has increased the chances of success.

(2) We were more flexible in managing the subretinal fluid. We prefer not to drain the subretinal fluid, but did not hesitate to evacuate it if necessary.

Lincoff, Baras, and McLean (1965) listed the indications for fluid drainage as follows: ballooned detachments, long-standing detachments, and posterior retinal breaks. Other indications for releasing the subretinal fluid include:
(I) Macular holes. These cannot be closed unless the retina around the hole is dry at the end of the operation, thus allowing the edges of the hole to touch the underlying choroid at the posterior pole of the eye.

(2) Co-existing glaucoma. The association of openangle glaucoma with rhegmatogenous retinal detachment is not rare (Linnér, I966; Schwartz, 1973). We noted that attempts at buckling without drainage of the subretinal fluid in these cases is resisted by a high intraocular pressure, so that the height of the buckle is insufficient to close the retinal breaks. Furthermore, if the fluid is not released, the intraocular pressure rises to high levels, thus compromising the patency of the central retinal artery.

(3) Co-existing retinitis pigmentosa. We had two cases of retinitis pigmentosa associated with rhegmatogenous retinal detachment. Absorption of the subretinal fluid is delayed in such cases by the morbid state of the choroidal vessels.

One possible disadvantage of silastic rod encirclement, is forward displacement of the irislens diaphragm if the globe is opened for an intraocular operation at some later date. This complication was seen in one of our patients who had a silastic rod encirclement and required cataract extraction 6 months later. Immediately after making the corneo-scleral section, the silastic rod recoiled by virtue of its inherent elasticity. The iris and lens prolapsed into the wound and the cataract extraction was attended by vitreous loss. This complication might have been avoided if the encircling rod had been cut before the cataract extraction.

\section{Conclusion}

Silastic rod encirclement, combined if necessary with additional circumferential or meridional silastic implants to obtain a more marked buckling effect at the site of a large or post-equatorial retinal break, has appreciably increased the chances of success of surgery for retinal detachment. Further indications for the release of subretinal fluid are described. The operation is easy to perform and the operative hazards few. Trauma to the ocular tissues in the primary operation is slight and this renders easy any subsequent operation which may be required.

\section{References}

CHAWLA, H. B. (197I) Brit. 7. Ophthal., 55, 331

Lincoff, H. A., Baras, I., and mCleAN, J. (1965) Arch. Ophthal. (Chicago), 73, i6o

LINNÉR, E. (1966) Acta ophthal. (Kbh.), Suppl. 84, p. Iо I

MORTADA, A. (1972) Brit. F. Ophthal., 56, 840

schwartz, A. (1973) Amer. F. Ophthal., 75, 205 